\section{Well-Managed Care}

Looking for a better healthcare provider? Consider self-analysis. The best system in the world, hands down, has many layers, thousands of workers, and is an incredible microman-

ager to boot-your immune system. From its innate to its adaptive defense strategies, the immune system is your body's main protection against a cold, cruel world. Complexity is no barrier to providing excellent healthcare, as a perusal of topics at Interhealth Molecular Immunology quickly reveals. The site's hierachical, hyperlinked headings span the entirety of immunology, thus tackling a complicated topic head on. In doing do, Molecular Immunology educates newbies as much as it aids researchers. Besides a primary focus on human immunology, there's also a considerable body of information on the immune systems of insects, mice, and even plants.

www.mi.interhealth.info

\section{Epilepsigenetics}

Epilepsy can be a debilitating medical condition. Affecting about $3 \%$ of the population, the affliction is characterized by several different forms of seizures that usually begin in adolescence. Epileptic seizures can arise from many causes, including injury, infection, and other brain trauma. What many people may not know, however, is that symptoms of the disease have been demonstrated in organisms as simple as nematodes. This evolutionary aspect of epilepsy provides researchers with important opportunities for understanding its genesis and developing treatments. Enter CarpeDB, the epilepsy database hosted at the University of Alabama, whose aim is exposing the disease's underlying genetics. At the web site, visitors can retrieve information by gene name, chromosome, description, keyword, or species. Returned records contain the massively hyperlinked gene information (NCBI, ENTREZ, LocusLink, Pfam, Swiss-Prot, UniProt, and others) now so commonly available for many genes.

www.carpedb.ua.edu

\section{Tuning Immunity}

Looking for a better understanding of how the immune system works its magic? Check out the Cytokine Family Database and learn about this fascinating class of polypeptides/glycopolypeptides and their associated cellular receptors. Often described as "hormones of the immune system," cytokines help to regulate cellular replication and differentiation by activation of organismal defenses. Cytokines help to control immune cells and regulate secretion of antibodies or even other cytokines. Sharing many properties of hormones and growth factors, cytokines are implicated in everything from primary and secondary immune responses. The site's offerings link to important cytokine information contained in other databases, such as $\mathrm{NCBI} / \mathrm{GenBank}{ }^{\circledR}$, UniGene, and others.

\section{cytokine.medic.kumamoto-u.ac.jp}

\section{CDNAs In Toto}

Once the bane of RNA workers, cDNAs are essential substrates for expressing eukaryotic messages in prokaryotic cells. The technology for making full-length versions of these important templates has improved to the point that today they are almost commodities available for trading. Through the Mammalian Gene Collection (MGC) site at the NIH, for example, full-length cDNAs for over 25,000 nonredundant human, mouse, and rat genes can now be purchased. All of the sequences have been deposited in GenBank ${ }^{\circledR}$ and are available for researchers to use. In addition, full-length cDNA collections from at least two other model organisms (Xenopus and Zebrafish) are, in the familiar words of the web, "under construction."

\section{Virtual Life for Real}

It's hard to imagine what molecular biology would be like today if not for information from Escherichia coli. The framework for much groundbreaking molecular research, this tiny cell is being positioned to become the first living system to leap from its native environment to the computer. Virtual life has long been a fascination for science fiction writers and computer programmers, but new modelers, systems biologists, are entering the picture in a big way via Project Cybercell. This mammoth effort will combine molecular structure/function data from genomics and proteomics with the mathematics/kinetics of biochemistry. Using computational/ biological methods, scientists hope to more accurately mimic life and obtain new perspectives on life and develop alternative strategies for combating infection. Can biocomputing emulate life? Stay tuned.

\section{www.projectcybercell.ca}

